Draft Version OCtOBer 1, 2018

Preprint typeset using $\mathrm{IAT}_{\mathrm{E}} \mathrm{X}$ style emulateapj v. 5/2/11

\title{
GRAVITATIONAL WAVES FROM THE COLLISION OF TIDALLY DISRUPTED STARS WITH MASSIVE BLACK HOLES
}

\author{
WILLIAM E. EAST \\ Kavli Institute for Particle Astrophysics and Cosmology, Stanford University, SLAC National Accelerator Laboratory, Menlo Park, CA \\ 94025, USA \\ Draft version October 1, 2018
}

\begin{abstract}
We use simulations of hydrodynamics coupled with full general relativity to investigate the gravitational waves produced by a star colliding with a massive black hole when the star's tidal disruption radius lies far outside of the black hole horizon. We consider both main-sequence and white-dwarf compaction stars, and nonspinning black holes, as well as those with near-extremal spin. We study the regime in between where the star can be accurately modeled by a point particle, and where tidal effects completely suppress the gravitational wave signal. We find that nonnegligible gravitational waves can be produced even when the star is strongly affected by tidal forces, as well as when it collides with large angular momentum. We discuss the implications that these results have for the potential observation of gravitational waves from these sources with future detectors.
\end{abstract}

Subject headings: black hole physics - gravitation - gravitational waves

\section{INTRODUCTION}

One exciting prospect for upcoming transient surveys is the possibility of detecting numerous tidal disruption events resulting from a star being pulled apart by a massive black hole $(\mathrm{BH})$ and then accreted. There have already been a number of candidate observations of such events across the electromagnetic spectrum (Bade et al. 1996, Komossa \& Greiner 1999, Gezari et al. 2003, 2006. 2008, Cappelluti et al. [2009, van Velzen et al. 2011; Bloom et al. 2011; Levan et al. 2011; Zauderer et al. 2011: Cenko et al. 2012a b: Gezari et al. 2012), and with the large number of expected future observations (van Velzen et al. 2011), there is hope that we will be able use such events to probe the strong-field gravity of massive BHs. These transients could occur not only for mainsequence stars falling into supermassive $\mathrm{BHs}$, but also possibly for white dwarfs falling into intermediate-mass BHs (Irwin et al. 2010 Krolik \& Piran 2011, Shcherbakov et al. 2013). However, complementing the events that produce these electromagnetic transients, there will be a population of stars launched on orbits without enough angular momentum to avoid being swallowed by the $\mathrm{BH}$. To get a rough idea of the relative rates, we can assume that the stars filling the $\mathrm{BH}$ loss cone have negligible orbital energy compared to their kinetic energy when orbiting near the $\mathrm{BH}$, and that the differential rate $d \Gamma$ of stars with specific orbital angular momentum $\tilde{L}$ obeys $d \Gamma \propto d\left(\tilde{L}^{2}\right)$ Frank \& Rees 1976, Kesden 2012), which holds in the so-called "pinhole" regime. For a nonspinning BH, parabolic orbits with $\tilde{L}<\tilde{L}_{\text {cap }}=4 M_{\mathrm{BH}}$ (where $M_{\mathrm{BH}}$ is the mass of the $\mathrm{BH}$ and we use geometric units $G=c=1$ throughout, unless otherwise stated) fall into the $\mathrm{BH}$ horizon. If we take the Newtonian estimate for the tidal disruption radius $r_{T}=R_{*}\left(M_{\mathrm{BH}} / M_{*}\right)^{1 / 3}$ for a star of mass $M_{*}$ and radius $R_{*}$, then the angular momentum of a Newtonian parabolic orbit with a periapse equal to $r_{T}$ is $\tilde{L}_{T}^{2}=2 M_{\mathrm{BH}}^{4 / 3} R_{*} / M_{*}^{1 / 3}$. Hence, the proportion of

weast@stanford.edu direct captures to those where the star is disrupted, but not captured, is given by $f=\left(\tilde{L}_{T}^{2} / \tilde{L}_{\text {cap }}^{2}-1\right)^{-1}$, where $\tilde{L}_{\text {cap }}^{2} / \tilde{L}_{T}^{2}=8\left(M_{\mathrm{BH}} / M_{*}\right)^{2 / 3}\left(M_{*} / R_{*}\right)$. Thus, for a supermassive $\mathrm{BH}$ with $M_{\mathrm{BH}}=10^{6} M_{\odot}$ and a star with solar mass and compaction, $M_{*} / R_{*}=2 \times 10^{-6}$, the ratio of collisions to disruption events is $\sim 0.2$.

A star colliding with a massive $\mathrm{BH}$ will most likely not produce a bright, fallback accretion-powered electromagnetic transient. However, such an event can produce gravitational waves (GWs). Gravitational waves can also be produced by a star as it passes through periapse and is disrupted in a nonmerging encounter (Kobayashi et al. 2004: Haas et al. 2012 Cheng \& Evans 2013), or potentially by the rebound of a tidally compressed star (Guillochon et al. 2009. Stone et al. 2013). Gravitational waves produced by white dwarts undergoing strong tidal interactions and mass transfer while orbiting massive BHs have also been considered (Zalamea et al. 2010; MacLeod et al. 2014). However, such signals will, in general, be weaker than those resulting from collisions. In addition, the merger-ringdown signal from a collision event, if detected, directly reveals the mass and spin of the $\mathrm{BH}$ (Berti et al. 2006). The ringdown frequency of supermassive BHs with masses $M_{\mathrm{BH}} \sim 10^{5}-10^{8} M_{\odot}$ falls in the frequency band of a space-based LISA-like GW instrument, while $\mathrm{BHs}$ with masses $M_{\mathrm{BH}} \lesssim 10^{3} M_{\odot}$ fall in the band of LIGO (Abramovici et al. 1992), KAGRA (Somiya 2012), and other similar ground-based detectors.

However, there are several reasons such GW signals may be difficult to detect at large distances. To begin with, for an extreme-mass-ratio system the amplitude of the GW is proportional to the mass ratio (for fixed total mass). For extreme-mass-ratio inspirals of compact objects, considered a promising source for a LISA-like GW detector (Amaro-Seoane et al. 2007), the system merges slowly through gravitational radiation reaction, and hence spends many wave cycles orbiting the $\mathrm{BH}$. However, in the collision case, the timescale of the merger 
is just set by the mass of the $\mathrm{BH}$ and is much shorter. Perhaps more importantly for the viability of these signals, there is the issue that as a star is pulled apart by tidal forces, and its radius becomes comparable to that of the $\mathrm{BH}$, the gravitational radiation it produces will be suppressed compared to a point particle of the same mass (Haugan et al. 1982).

It is the purpose of this paper to investigate the details of the GW signal produced by a star undergoing tidal disruption while colliding with a massive BH. For stars whose radii are small compared to that of the $\mathrm{BH}$, and whose tidal disruption radius does not lie outside the $\mathrm{BH}$ horizon, we expect the gravitational waveform to be essentially identical to that of an equivalent point particle. However, when tidal forces disperse the star's mass enough so that the different mass elements of the star no longer excite gravitational radiation from the $\mathrm{BH}$ coherently, the signal becomes significantly suppressed. In Haugan et al. (1982), this was demonstrated by estimating the suppression of gravitational-wave energy due to incoherence for the head-on collision of a star with a $\mathrm{BH}$ using $\mathrm{BH}$ perturbation theory. The star was modeled with a spherical dust cloud undergoing free fall from the nominal tidal disruption radius of the star. In the limit that the tidal radius goes to infinity, no gravitational waves are produced.

In this work, we are interested in studying the details of GW production in the intermediate regime, between when the star can be treated as a point particle and tidal forces can be ignored and when GW production is minimal because of strong tidal forces (such as for the spherical dust cloud free-falling from infinity). We make use of recently developed techniques in the simulation of full general relativity coupled with hydrodynamics in the extreme-mass-ratio regime in order to take into account both the star's self-gravity and gravitational perturbation, as well as the strong-field effects of the $\mathrm{BH}$ on the hydrodynamics of the star. These methods allow us to self-consistently compute the full GW signal, including the ringdown of the perturbed $\mathrm{BH}$. Besides considering head-on collisions with nonspinning and spinning $\mathrm{BHs}$, we also consider collisions with nonzero angular momentum. Angular momentum can significantly enhance the amplitude of the GW produced. In fact, for a point particle, the energy in GWs formally diverges as $\tilde{L} \rightarrow \tilde{L}_{\text {cap }}$ since the corresponding geodesic circles the $\mathrm{BH}$ an infinite number of times at the innermost stable orbit (thus signaling that back-reaction effects must be taken into account). For the star-BH system, it is thus of interest to investigate when finite size effects will be important in order to determine whether these events might eventually constitute viable GW sources.

The remainder of this paper is as follows. We outline our numerical methods for accurately evolving the Einstein-hydrodynamic equations in Section 2, and describe the different cases we simulate in Section 3. We present the results of these simulations in Section 4 where we find that nonnegligible GW radiation can be produced well into the regime where tidal effects are important, both for head-on collisions, as well as those with significant angular momentum. In Section 5 we compare the simulations to a simple model based on geodesics which we find explains the main features of the simula- tions well and can be used to generalize the results. We briefly comment on the detectability of these GW signals in Section 6, and discuss the implications of these results and conclude in Section 7.

\section{NUMERICAL METHODS AND SETUP}

In order to study the GW signal produced by the collision of a star with a massive $\mathrm{BH}$, we perform simulations of hydrodynamics coupled with the Einstein-field equations using the code described in East et al. (2012a). To accurately evolve the star's small contribution to the spacetime metric, without it being washed out by truncation error from the dominant $\mathrm{BH}$ solution, we use the background error subtraction technique (BEST) developed in East \& Pretorius (2013). BEST exploits the fact that the isolated $\mathrm{BH}$ solution is a known, exact solution to the field equations, in order to explicitly subtract out its contribution to the truncation error for our particular numerical scheme. This method was used to evolve star-BH systems with mass ratios up to $8 \times 10^{6}$ in East \& Pretorius (2013). To minimize the advection error, we also perform the simulation in the rest frame of the star as in East \& Pretorius (2013). For the subset of cases considered below that are head-on collisions, we use the axisymmetry of the setup to restrict the computational domain to the half-plane using a modified version of the Cartoon method (Alcubierre et al. 2001) as described in Pretorius (2005).

We model the stars as Tolman-Oppenheimer-Volkoff solutions with a gamma law equation state with $\Gamma=5 / 3$ (discussed in Section 3). The initial star profiles are taken to be polytropic, though we allow for shock heating during the evolution. We begin the simulation with the star outside its tidal disruption radius (at $d=200 M_{\mathrm{BH}}$ and $100 M_{\mathrm{BH}}$ for the head-on main-sequence star and white dwarf cases, respectively, and $d=1.2 r_{T}$ for the three-dimensional cases) and choose the initial velocity of the star based on the geodesic of the $\mathrm{BH}$ spacetime with the corresponding orbital energy and angular momentum. For all of the cases considered below, we assume that the star has zero orbital energy, i.e., it is marginally unbound.

For the $\mathrm{BH}$ solution, we use harmonic coordinates (Cook \& Scheel 1997) for the nonspinning cases below; however, for the spinning cases, we use Kerr-Schild coordinates since this solution is better behaved in the near-extremal-spin limit. Consistent initial data for the combined BH-star system is found by solving the constraint equations using the code described in East et al. (2012b).

In order to measure the GW signal, we use the metric to calculate the Newman-Penrose scalar $\Psi_{4}$ at a radius of $r=100 M_{\mathrm{BH}}$ from the center of mass. We decompose $\Psi_{4}$ into spin weight -2 spherical harmonics labeled by $\ell$ and $m$. We note that for axisymmetric cases only the $m=0$ components are nonzero.

The simulations make use of adaptive mesh refinement based on truncation error. Most of the simulations described here have up to eight levels of mesh refinement with a 2:1 refinement ratio and a resolution of $\approx 0.01 M_{\mathrm{BH}}$ on the finest level (for the one $M_{\mathrm{BH}} / M_{*}=$ $2 \times 10^{6}$ case, we add one additional level) and a resolution of $1.2 M_{\mathrm{BH}}$ in the wave zone. In Figure 1, we also show the gravitational waveforms from simulations 
with higher $(1.5$ times $)$ and lower $(\approx 0.8$ times $)$ resolution to demonstrate the expected second-order convergence and to indicate the level of truncation error. We also demonstrate how well the simulation balances selfgravity against pressure for an isolated main-sequence star solution in Figure 2, where we compare the initial solution to one evolved with the lower resolution for $10 R_{*} / c_{s}$, where $c_{s}$ is the sound speed at the center of the star. (For comparison, for the simulation with the main-sequence star and $M_{\mathrm{BH}} / M_{*}=10^{6}$, the radial freefall time from the initial separation of $d=200 M_{\mathrm{BH}}$ used here is $\left.\approx 4 R_{*} / c_{s}.\right)$ For the $3 \mathrm{D}$ simulations, the resolution is equivalent to the lower value of resolution, and since the same coordinates, etc., are used, the truncation error is comparable.

\section{CASES}

We consider two different types of stars. To initially represent a main-sequence star, we choose the $\Gamma=5 / 3$ polytropic Tolman-Oppenheimer-Volkoff solution with compaction $M_{*} / R_{*}=2 \times 10^{-6}$ (equivalent to $\left.R_{*} \approx\left(M_{*} / M_{\odot}\right) R_{\odot}\right)$. To represent a white dwarf, we choose the $\Gamma=5 / 3$ polytropic solution with compaction $M_{*} / R_{*}=2.5 \times 10^{-4}$ (equivalent to $R_{*} \approx$ $\left.\left(M_{*} / M_{\odot}\right) 6 \times 10^{3} \mathrm{~km}\right)$. As described in Cook et al. (1994), for such polytropic solutions one has the freedom to choose any overall length scale (or equivalently, mass scale), though when considering the potential GW detectability in Section 6, we will consider different specific choices of physical mass.

Because of the computational expense of the 3D cases, we restrict most of our simulations to head-on collisions. We consider the head-on collisions of main-sequence stars with nonspinning BHs with mass ratios $M_{\mathrm{BH}} / M_{*}=1$, $0.5,0.25$, and $0.125 \times 10^{6}$. The main-sequence star has a nominal Newtonian tidal disruption radius of $r_{T} / M_{\mathrm{BH}}=$ $50\left(M_{*} / M_{\mathrm{BH}} \times 10^{6}\right)^{2 / 3}$. (We note that in geometric units $10^{6} M_{\odot}$ is equivalent to $\approx 1.5 \times 10^{6} \mathrm{~km}$ or $\approx 5$ s.) In order to probe any effects of $\mathrm{BH}$ spin, we also consider the collisions of main-sequence stars with $\mathrm{BHs}$ with dimensionless spin $a=0.99$, where the spin and collision axes are aligned. For the spinning cases, which have more high-frequency power, we consider a slightly higher range of mass ratios given by $M_{\mathrm{BH}} / M_{*}=2,1$, 0.5 , and $0.25 \times 10^{6}$ We also study the head-on collisions of a white dwarf with a nonspinning $\mathrm{BH}$ with mass ratios $M_{\mathrm{BH}} / M_{*}=4,2,1$, and $0.5 \times 10^{3}$. The white dwarf has a nominal Newtonian tidal disruption radius of $r_{T} / M_{\mathrm{BH}}=40\left(M_{*} / M_{\mathrm{BH}} \times 10^{3}\right)^{2 / 3}$. With these parameters, for the same value of the tidal disruption radius in units of $\mathrm{BH}$ mass (i.e., the same value of $r_{T} / M_{\mathrm{BH}}$ ), the white dwarf's size compared to the $\mathrm{BH}$ (i.e., $R_{*} / M_{\mathrm{BH}}$ ) will be larger than for main-sequence stars. Hence, we can anticipate that GW suppression due to decoherence will happen at somewhat smaller values of $r_{T} / M_{\mathrm{BH}}$ for the white dwarf compared to the main-sequence star.

Finally, we consider two of the more computationally expensive nonzero-angular-momentum collisions of a main-sequence star and a nonspinning $\mathrm{BH}$ with $M_{\mathrm{BH}} / M_{*}=10^{6}$. These cases have reduced orbital angular momentums of $\tilde{L} / M_{\mathrm{BH}}=2$ and 3.5 , which should be compared to the maximum angular momentum for the
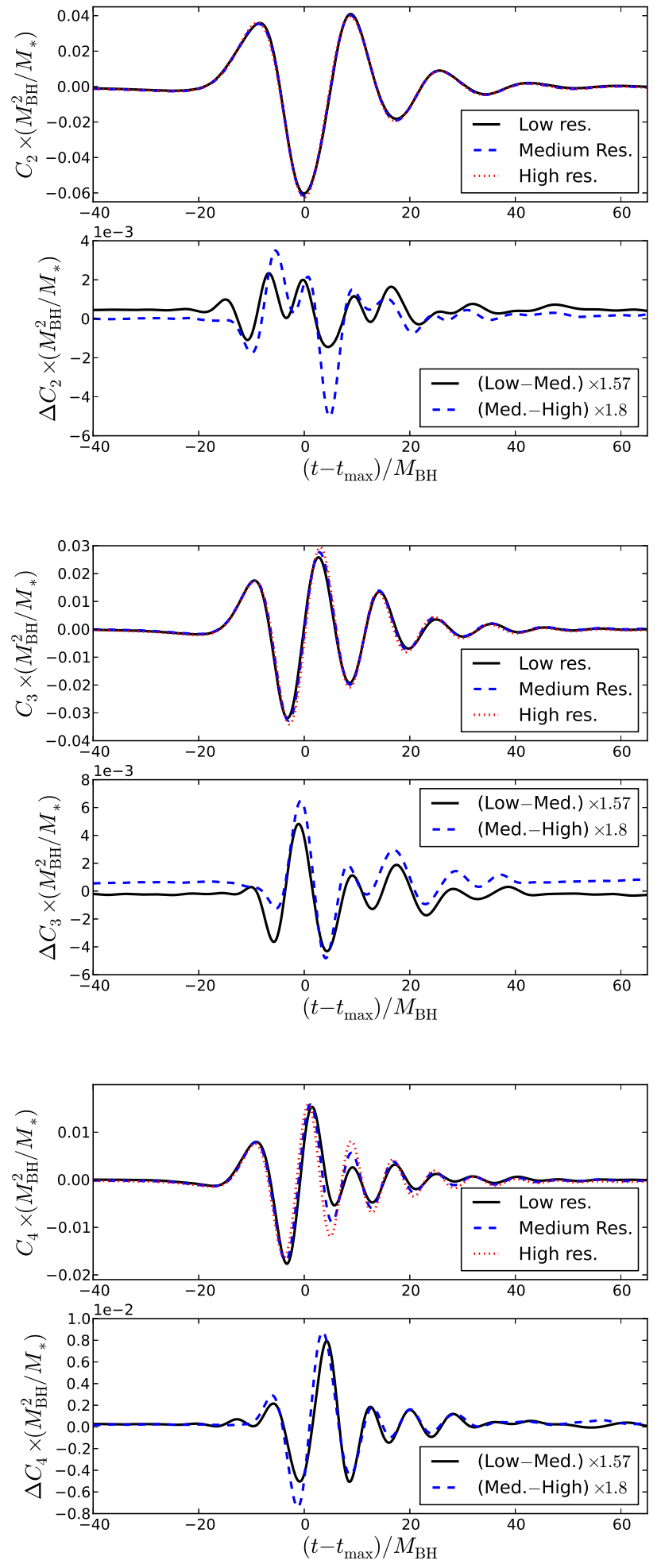

FIG. 1.- From top to bottom, the $\ell=2,3$, and 4 (with $m=0$ ) spin weight -2 spherical harmonics of $r \Psi_{4}$ from a nonspinning, $M_{\mathrm{BH}} / M_{*}=10^{6}$, head-on collision simulation performed at three resolutions. In each case, the lower panel shows the difference between the different resolutions, scaled assuming second-order convergence. 


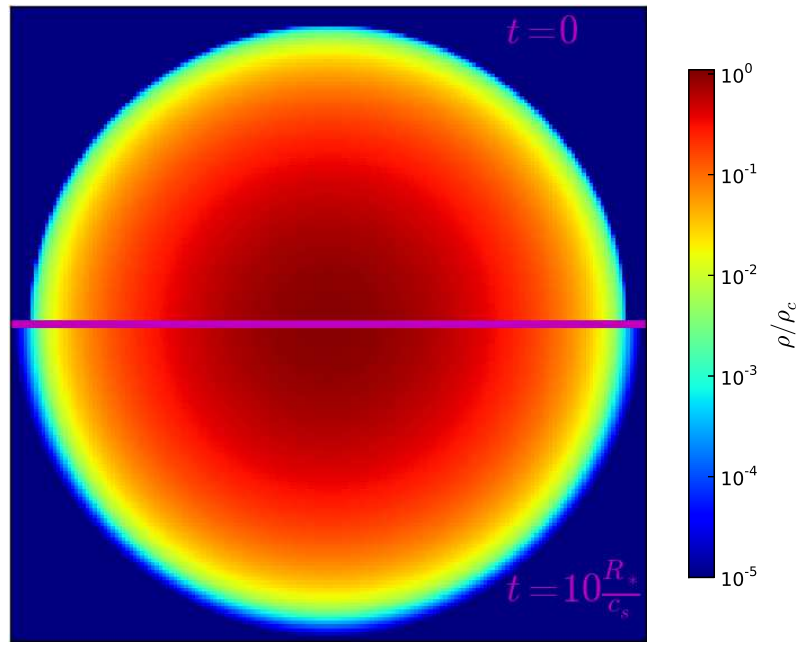

Fig. 2. - Snapshot of rest-mass density (in units of the initial central density of the star) for an isolated main-sequence star solution at $t=0$ (top) and after being evolved $10 R_{*} / c_{s}$ (bottom), where $c_{s}$ is the central sound speed, with resolution corresponding to approximately 40 cells across the radius of the star on the finest level. This illustrates how well the simulation can maintain an equilibrium solution at this resolution.

star to fall into the $\mathrm{BH}$ of $\tilde{L}_{\text {cap }} / M_{\mathrm{BH}}=4$. We do not consider the case with $\tilde{L}=\tilde{L}_{\text {cap }}$ because tidal pancaking makes these simulations more computationally challenging, though we will argue in Section 5 that decoherence is expected to strongly suppress the GW radiation in this case.

\section{RESULTS}

\subsection{Main-sequence Stars}

For star-BH collisions with low orbital angular momentum, the gravitational waveform will be dominated by the merger of the star with the $\mathrm{BH}$, followed by the quasi-normal mode (QNM) ringing of the perturbed $\mathrm{BH}$ post-merger. In Figure 3, we show the first three spherical harmonics of $\Psi_{4}$ from the head-on collisions of mainsequence stars with a $\mathrm{BH}$, scaled with the mass ratio so that they would agree in the point-particle limit. For $M_{\mathrm{BH}} / M_{*} \geq 10^{6}$, the waveform agrees to within a few percent (roughly comparable to the truncation error - see Figure 11 with the point-particle prediction, which gives an energy in GWs of $E_{\mathrm{GW}}=0.0104 M_{*}^{2} / M_{\mathrm{BH}}$ (Davis et al. 1971). Below this mass ratio, the gravitational radiation begins to be suppressed. However, even at $M_{\mathrm{BH}}=2.5 \times 10^{5} M_{*}$, the amplitude of the dominant $l=2$ mode is still $\sim 1 / 3$ of the point-particle value. We emphasize that the waveforms are shown scaled by the mass ratio, and, for a fixed value of $M_{\mathrm{BH}}$, the amplitude of the $M_{\mathrm{BH}}=2.5 \times 10^{5} M_{*}$ simulation is in fact larger than the $M_{\mathrm{BH}}=10^{6} M_{*}$. In the lower panels of Figure 3 . we can see that the higher harmonics are slightly more suppressed at smaller mass ratios, which can be related to the fact that higher $\ell$ QNMs are at slightly higher frequencies (e.g., the least-damped QNM mode frequencies are $\omega_{\mathrm{QNM}} M_{\mathrm{BH}}=0.37,0.60$, and 0.81 for $l=2,3$, and $4(m=0)$, respectively (Berti et al. 2006) and are thus more easily suppressed when the differential timescale over which the different mass elements of the star merge with the $\mathrm{BH}$ is comparable to $M_{\mathrm{BH}}$.

In the top panel of Figure 4, we show the GW power spectrum for the same cases as in Figure 3. The lowfrequency GW power comes from the star falling into the $\mathrm{BH}$ at large distances, while the spectrum peaks at around the BH's dominant QNM frequency, and falls off rapidly above. From this figure, it is apparent that with decreasing mass ratio, and hence increasing tidal radius, more and more of the higher frequency power is suppressed. This results in the peak power shifting to lower frequencies.

We also perform the same simulations as above, but with a $\mathrm{BH}$ with dimensionless spin $a=0.99$. We note that in order to preserve the axisymmetry of the simulation, we consider the case where the collision axis and the spin axis are aligned. In the point-particle limit, this setup results in 1.65 times as much energy in GWs as the nonspinning case (Nakamura \& Sasaki 1982 ), though only 0.4 times as much energy as in the case where the spin and collision axes are perpendicular (Kojima \& Nakamura 1983b). In Figure 5 and in the middle panel of Figure 4. we show, respectively, the gravitational waveforms and power spectra for these simulations. These figures illustrate that the merger-ringdown of a highly spinning $\mathrm{BH}$ has additional power at higher frequencies. The behavior with mass ratio is similar to the nonspinning case, though in this case the GW energy in the $M_{\mathrm{BH}} / M_{*}=10^{6}$ case is slightly more suppressed at $\approx 85 \%$ of the point-particle prediction. Comparing to the higher mass ratio $M_{\mathrm{BH}} / M_{*}=2 \times 10^{6}$ case, we can see that the former case is missing energy at higher frequencies, and that, in particular, the higher $\ell$ modes (which resonant at higher frequencies) are noticeably suppressed.

Coherent merger-ringdown GW signals are possible not only for head-on star-BH collisions, but also for those with nonnegligible angular momentum. In Figure 6. we show the dominant $\ell=m=2$ component of the waveform for two cases with reduced angular momentum $\tilde{L} / M_{\mathrm{BH}}=2$ and 3.5. These two cases have $E_{\mathrm{GW}} M_{\mathrm{BH}} / M_{*}^{2} \approx 0.039$ and 0.19 , respectively, which correspond to $\sim 90 \%$ and $\sim 75 \%$ of the point-particle prediction. With increasing angular momentum, the waveform amplitude increases and begins to show evidence of "whirling" behavior before plunging into the BH. Figure 7 shows snapshots of the star's fluid density from these cases shortly before collision. As can be seen, with increasing angular momentum, the star also becomes more stretched out in the orbital plane, though denser at its center due to being compressed in the perpendicular direction. However, for $\tilde{L} / M_{\mathrm{BH}}=3.5$, the star's size is still comparable to the BH's. In Section 5, we estimate how much closer to $\tilde{L}_{\text {cap }}$ the star may be able to coherently excite GWs.

\subsection{White Dwarfs}

For the collision of white dwarfs with massive BHs, we find qualitatively similar behavior to the main-sequence star cases around the transition from mass ratios that can be treated as close to point particles, to those where decoherence is important. This can be seen in Figure 8 and the bottom panel of Figure 4 When scaled ap- 

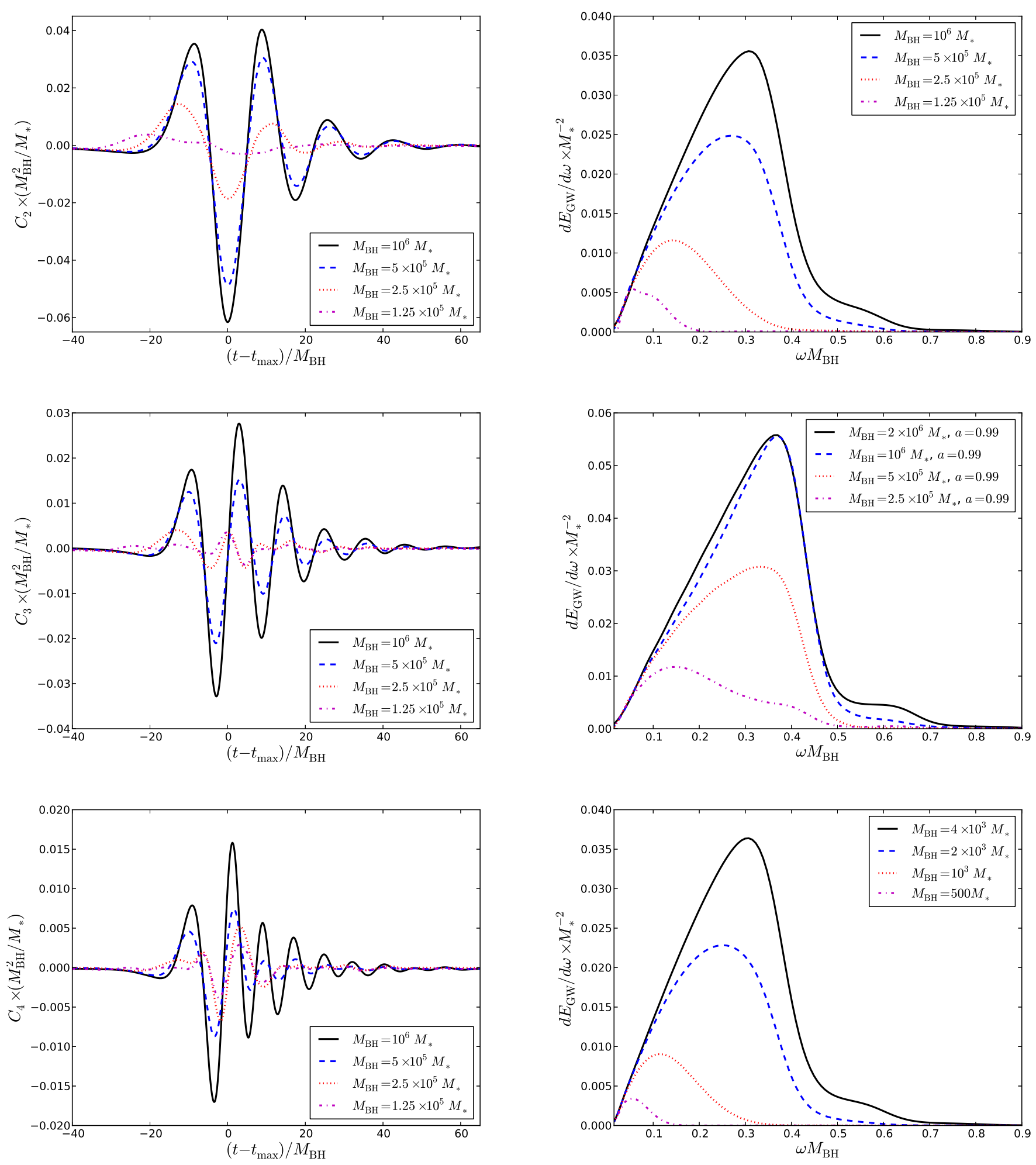

Fig. 3.- From top to bottom, the $\ell=2,3$, and 4 (with $m=0$ ) spin weight -2 spherical harmonics of $r \Psi_{4}$ for the head-on collision of a main-sequence star and a nonspinning $\mathrm{BH}$ for various mass ratios. The amplitude has been scaled so that the curves would align in the point-particle limit. Here $t_{\max }$ is the time when the $l=2$ amplitude is maximum for the largest mass ratio case.

FIG. 4.- Gravitational wave power spectrum (scaled so that the curves would agree in the point-particle limit) from the head-on collision of a star and a BH. From top to bottom, we show results for a main-sequence star and a nonspinning $\mathrm{BH}$, a main-sequence star and an $a=0.99 \mathrm{BH}$, and a white dwarf and a nonspinning $\mathrm{BH}$, all for various mass ratios. 

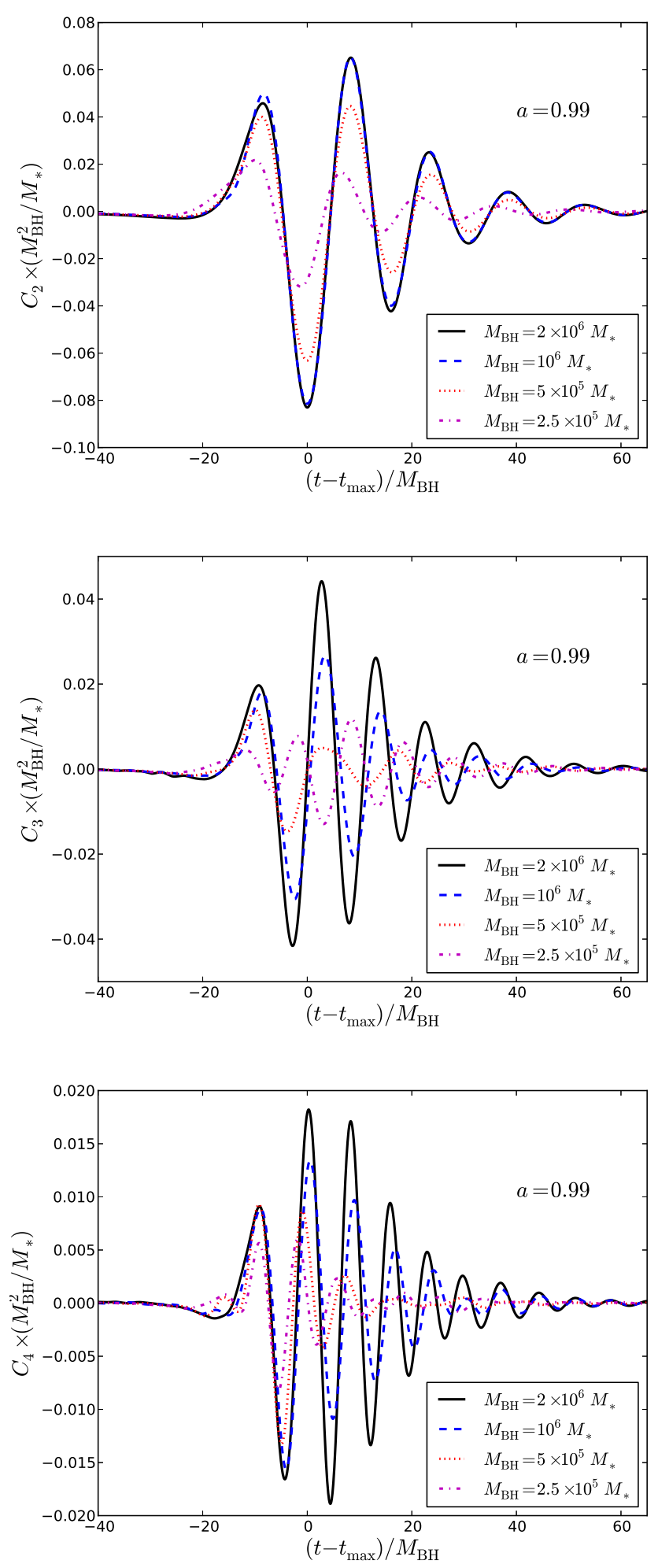

Fig. 5.- Same as Figure 3 but for the head-on collision of a main-sequence star and a $\mathrm{BH}$ with dimensionless spin $a=0.99$.
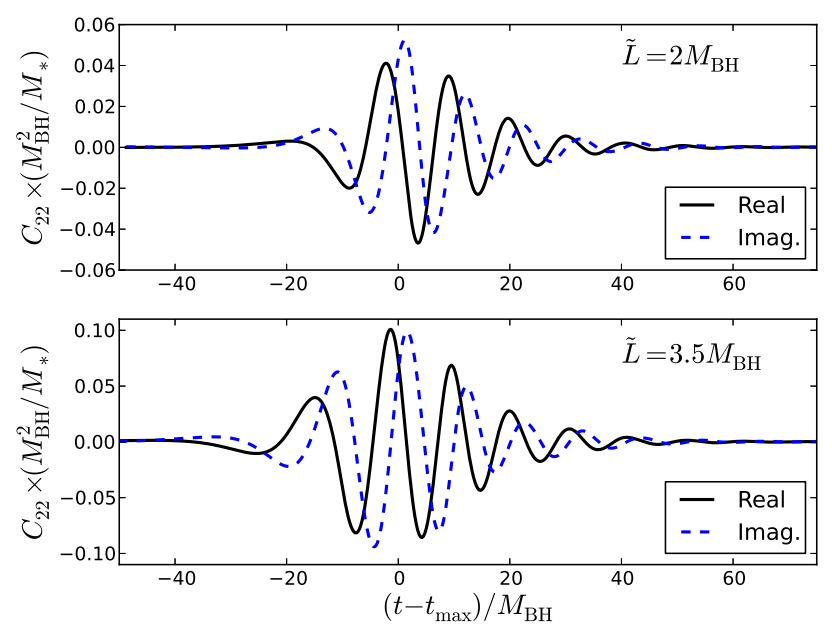

FIG. 6. $-\ell=m=2$ spin weight -2 spherical harmonic of $r \Psi_{4}$ for the collision of a main-sequence star and a $\mathrm{BH}$ with $M_{\mathrm{BH}} / M_{*}=$ $10^{6}$ and $\tilde{L} / M_{\mathrm{BH}}=2$ (top) and 3.5 (bottom).

propriately by the mass ratio, the waveform from the $M_{\mathrm{BH}} / M_{*}=4 \times 10^{3}$ case is almost identical to the $M_{\mathrm{BH}} / M_{*}=10^{6}$ main-sequence star case (and hence also the point-particle prediction). For smaller mass ratios, this is no longer true. The $M_{\mathrm{BH}} / M_{*}=2 \times 10^{3}$ and $M_{\mathrm{BH}} / M_{*}=10^{3}$ cases have, respectively, $\approx 65 \%$ and $\approx 15 \%$ the energy in GWs (scaled by $M_{\mathrm{BH}}^{2} / M_{*}$ ) as the $M_{\mathrm{BH}} / M_{*}=4 \times 10^{3}$ case. Compared to the mainsequence star simulations, the suppression of GW energy due to finite size effects not only sets in at smaller mass ratios (since the white dwarf is more compact) but also at somewhat smaller values of the tidal disruption radius. As noted above, this is because for the white-dwarf compaction used here, the white dwarf's unperturbed size is comparable to the radius of the $\mathrm{BH}$ at smaller values of $r_{T}$. This can be seen in Figure 9 where we show snapshots of the white-dwarf density around the time of collision for the $M_{\mathrm{BH}} / M_{*}=2 \times 10^{3}$ and $4 \times 10^{3}$ cases.

\section{GEODESIC MODEL OF TIDAL DISRUPTION}

In order to compare to the results from the full simulations, and in order to generalize them to cases that were not run, we can construct a simple model based on geodesics of an isolated BH spacetime along the lines considered in Haugan et al. (1982) and Kesden (2012). Corresponding to a star with radius $R_{*}$ and tidal disruption radius $r_{T}$, we consider a set of equatorial geodesics parameterized by an angle $\theta$ with initial positions $(x, y)=\left(x_{c}+R_{*} \cos \theta, y_{c}+R_{*} \sin \theta\right)$ where $\sqrt{x_{c}^{2}+y_{c}^{2}}=r_{T}$ and all with the same initial velocity given by the trajectory of the star's center of mass at $r=r_{T}$. Then we can compute the time $\Delta t_{\text {geo }}$ it takes for these geodesics to cross the BH light ring compared to the center-of-mass geodesic. We ignore nonequatorial orbits since tidal forces will flatten the star in the direction perpendicular to the orbital plane. This simplistic model of course ignores the dynamics of the disruption process and assumes that the combined effects of the star's self-gravity and pressure support can be ignored 

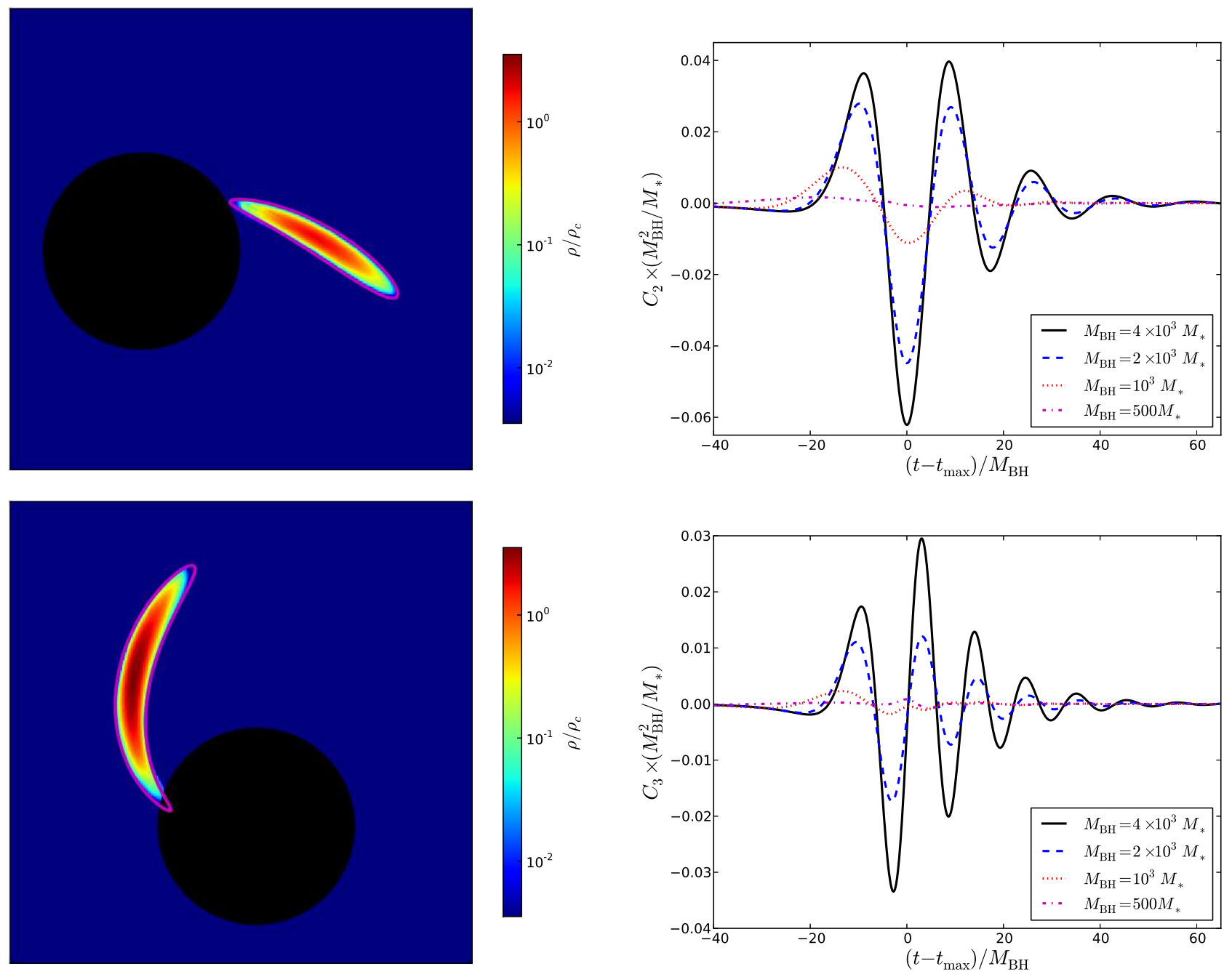

FIG. 7.- Snapshots of rest-mass density (in units of the initial central density of the star) in the orbital plane around the time that the star's center of mass crosses the BH's light ring from simulations with main-sequence stars, $M_{\mathrm{BH}} / M_{*}=10^{6}$, and $\tilde{L} / M_{\mathrm{BH}}=2$ (top), and 3.5 (bottom). Also shown is the position of the set of geodesics from the corresponding model described in Section 5 (magenta outline) at the same time as the simulation snapshot.

for $r \leq r_{T}$, while, for $r>r_{T}$, tidal effects can be completely ignored. It also does not capture the distribution of the star's mass, which, in general, will be centrally condensed. In addition, we note that the quantity $\Delta t_{\text {geo }}$ is coordinate dependent (here we use harmonic coordinates as in the simulations). Nevertheless, we find that this model captures the main features of the simulation results.

As an initial point of comparison, in Figures 7 and 9 . along with the density from the full simulation results at approximately the time the star's center of mass crosses the BH's light ring, we also include the curve made up by the positions of the set of geodesics for the corresponding parameters and time. We can see that in fact this curve matches the shape of the star quite well in all cases. This indicates that the model assumptions are fairly good approximations for these cases.

In Figure 10, we show the values of $\Delta t_{\text {geo }}$ computed for

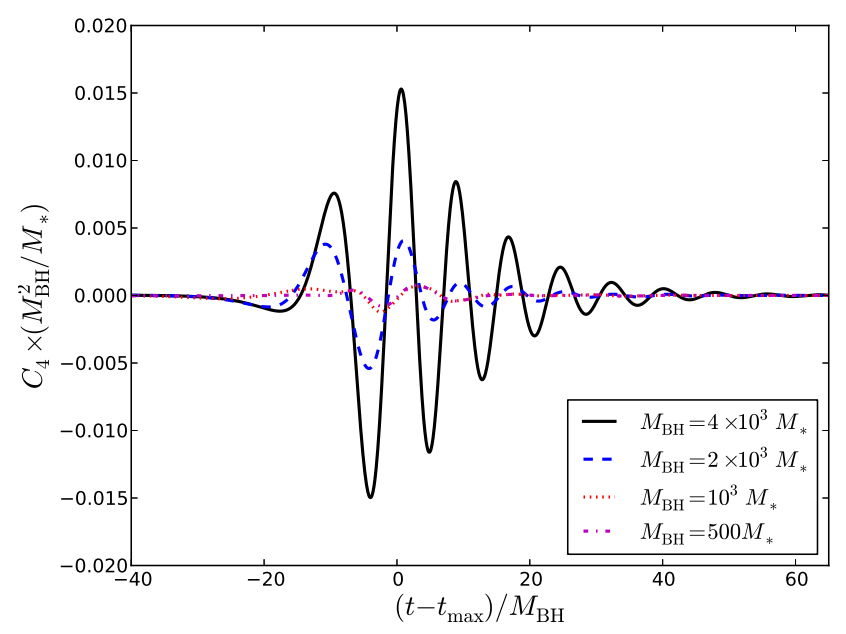

FIG. 8. - Same as Figure 3 but for the head-on collision of a white dwarf and a nonspinning $\mathrm{BH}$. 

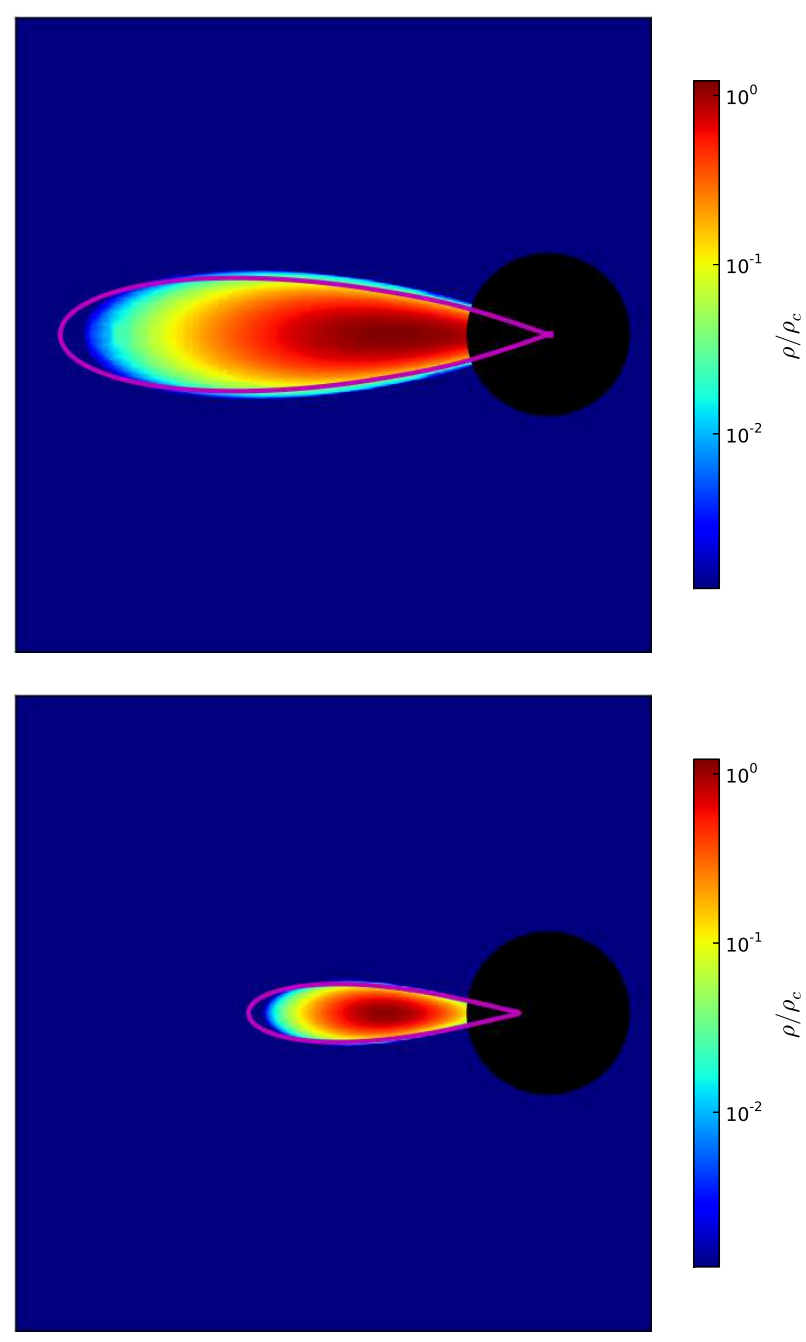

FIG. 9. - Same as Figure 7 but for the head-on collision of a white dwarf with a $\mathrm{BH}$ with mass $M_{\mathrm{BH}} / M_{*}=2 \times 10^{3}$ (top) and $M_{\mathrm{BH}} / M_{*}=4 \times 10^{3}$ (bottom).

different values of $R_{*}$ and $r_{T}$ corresponding to the model main-sequence star. Since for large mass ratios the GW power spectrum peaks at $\omega \sim 0.3 / M_{\mathrm{BH}}$ (Figure 4), we expect decoherence of the GW signal when $\left|\Delta t_{\text {geo }}\right| \gtrsim$ $\pi / \omega \sim 10 M_{\mathrm{BH}}$. The top panel of Figure 10 shows that for head-on collisions the $M_{\mathrm{BH}} / M_{*}=5 \times 10^{5}$ case lies below this threshold, while the $M_{\mathrm{BH}} / M_{*}=2.5 \times 10^{5}$ case exceeds it. This is fully consistent with the results from the full simulations where the former case had $\sim 70 \%$ of the point-particle prediction for GW energy, and the latter $\sim 25 \%$. The bottom panel indicates that in the $\tilde{L} / M_{\mathrm{BH}}=2$ and 3.5 cases with $M_{\mathrm{BH}} / M_{*}=10^{6}$, gravitational radiation should not be significantly suppressed, also consistent with what was found in the simulations. Furthermore, the geodesic model suggests that this should hold for $\tilde{L} / M_{\mathrm{BH}} \lesssim 3.75$. As $\tilde{L} \rightarrow \tilde{L}_{\text {cap }}$, part of the star will collide with the $\mathrm{BH}$, while part of it will go back out on a long (possibly unbound) orbit, as illustrated by the $\tilde{L} / M_{\mathrm{BH}}=3.9$ case in Figure 10 . Hence decoherence effects will be strong.

We also apply the geodesic model to white dwarf pa-

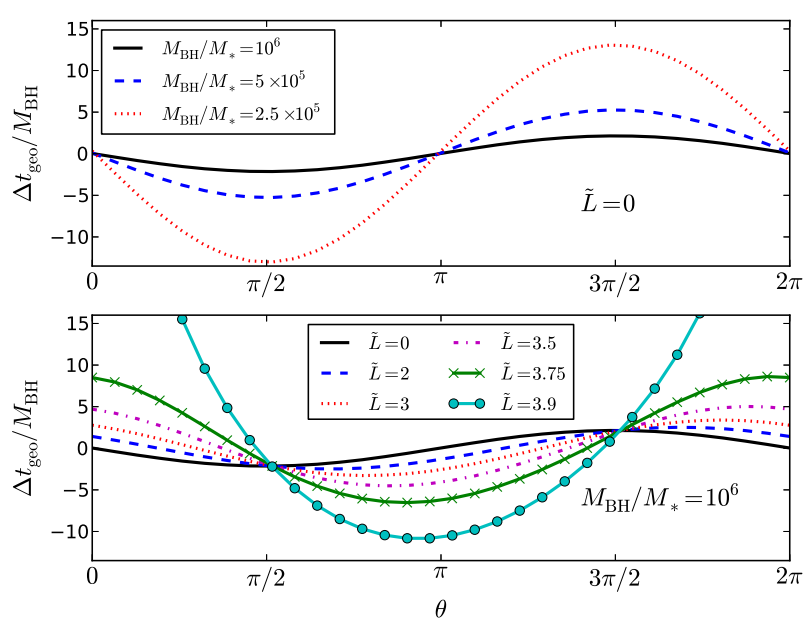

FIG. 10.- Relative difference in time for geodesics to cross the $\mathrm{BH}$ light ring for model parameters corresponding to a mainsequence star. The top panel corresponds to head-on collisions with different mass ratios, while the bottom panel corresponds to a $M_{\mathrm{BH}} / M_{*}=10^{6}$ mass ratio with different amounts of angular momentum.
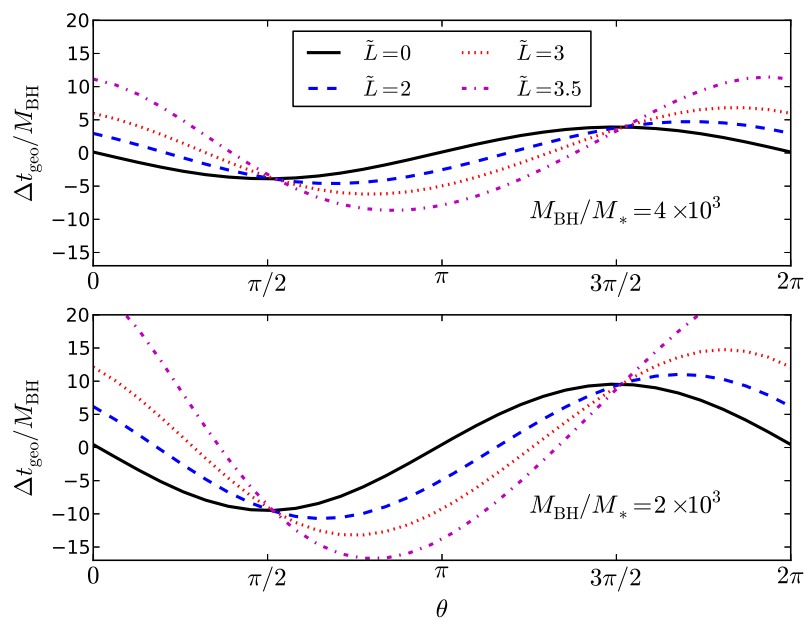

FIG. 11. - Relative difference in time for geodesics to cross the $\mathrm{BH}$ light ring for parameters corresponding to a white dwarf. The top panel and bottom panel have parameters corresponding to mass ratios of $M_{\mathrm{BH}} / M_{*}=4 \times 10^{3}$ and $M_{\mathrm{BH}} / M_{*}=2 \times 10^{3}$, respectively.

rameters to obtain the results shown in Figure 11. For $M_{\mathrm{BH}} / M_{*}=4 \times 10^{3}$ this indicates that decoherence should not set in for $\tilde{L} / M_{\mathrm{BH}} \lesssim 3.5$. For the $M_{\mathrm{BH}} / M_{*}=$ $2 \times 10^{3}$ case, the model indicates that a head-on collision should be right at the decoherence threshold (we recall that the simulation for this case had $\approx 65 \%$ of the pointparticle prediction for GW energy), but that the magnitude of $\Delta t_{\text {geo }}$ is only slightly increased for $\tilde{L} / M_{\mathrm{BH}} \leq 2$.

\section{PROSPECTS FOR DETECTION}

We can use the gravitational waveforms obtained from these simulations to estimate the distance at which future GW detectors might be able observe such events. To do 


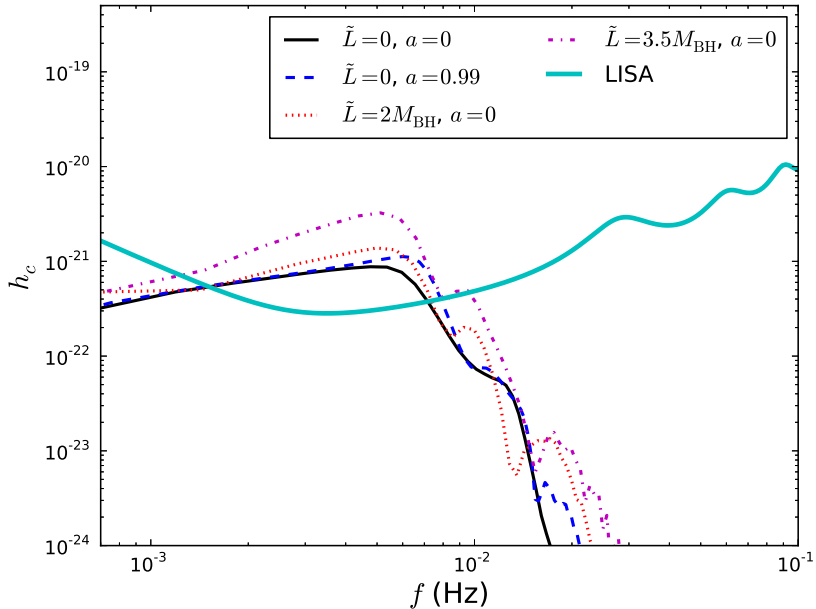

FIG. 12.- Characteristic GW strain for main-sequence star-BH collisions with $M_{*}=2 M_{\odot}$ and $M_{\mathrm{BH}}=2 \times 10^{6} M_{\odot}$ assumed to be observed with optimal orientation at a distance of $d=15 \mathrm{Mpc}$. For comparison, we also show a proposed LISA noise curve.

so, we need to choose an overall physical mass for the system to scale the results of the simulation. (We note that the choice $M_{*} / R_{*}=2 \times 10^{-6}$ for a main-sequence star is appropriate for $M_{*} \sim M_{\odot}$ and more massive stars will be slightly more compact, while less massive stars will be less compact, but we ignore that weak dependence here.) In Figure 12, we show the characteristic GW strain $h_{c}$ from the four simulations with $M_{\mathrm{BH}} / M_{*}=10^{6}$, assuming $M_{*}=2 M_{\odot}$ and an optimally oriented and located source at a distance of $15 \mathrm{Mpc}$. The characteristic strain is defined as $h_{c}=|\tilde{h}| f$ where $\tilde{h}$ is the Fourier transform of the strain and $f$ is frequency. For comparison, we also show a proposed LISA noise curve ${ }^{1}$ where for a noise power spectral density $S_{n}(f)$, we define the characteristic strain as $h_{n}=\sqrt{S_{n} f}$. With these definitions, the signal-to-noise of a match filtered signal is given by $\mathrm{S} / \mathrm{N}=2\left[\int h_{c} / h_{n} d \ln (f)\right]^{1 / 2}$, i.e., is given by integrating the ratio of characteristic strain signal- to-noise over a logarithmic frequency interval. In Figure 12, we see that BHs with spin have an additional signal at high frequencies compared to nonspinning $\mathrm{BHs}$, and that signals from collisions with more angular momentum are significantly stronger. For example, for $\tilde{L} / M_{\mathrm{BH}}=3.5, \mathrm{~S} / \mathrm{N} \approx 18$, while the $\tilde{L} / M_{\mathrm{BH}}=2$ and head-on case have, respectively, $\sim 0.5$ and 0.4 the $\mathrm{S} / \mathrm{N}$ of the head-on case for these parameters. Non-head-on collisions with spinning BHs or collisions with somewhat higher $\tilde{L}$ than considered here (the results of Section 5 suggest $\tilde{L} / M_{\mathrm{BH}} \leq 3.75$ will not be strongly suppressed) could potentially produce stronger signals. The signal-to-noise is not extremely sensitive to physical mass in this range. Scaling the results to $M_{*}=4 M_{\odot}$ instead of $M_{*}=2 M_{\odot}$ increases

\footnotetext{
${ }^{1}$ We use the LISA noise curve from Larson (accessed June 2014) that includes both pointing and shot noise, but remove the all-sky and polarization averaging factor as in Berti et al. (2006). We also note that the proposed eLISA design is less sensitive at lower frequencies than the original LISA design (Amaro-Seoane et al. 2013).
}

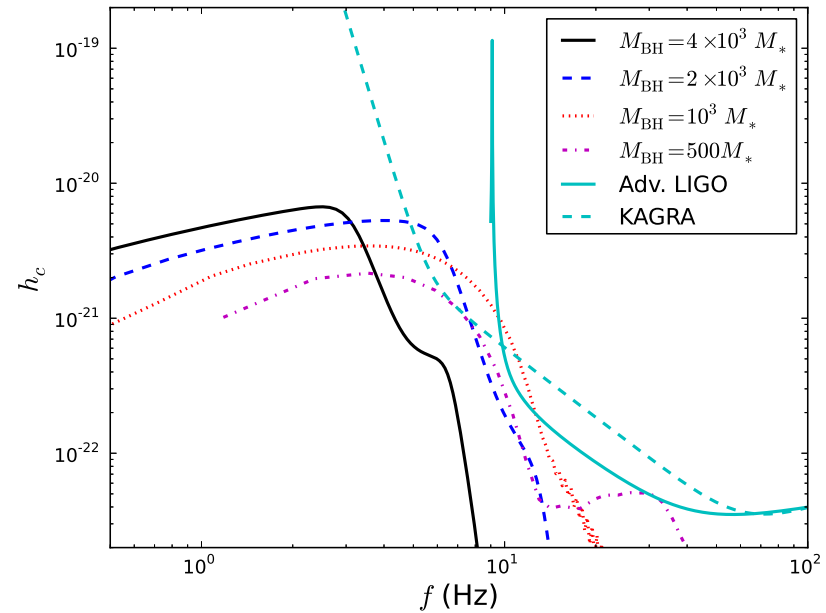

FIG. 13.- Characteristic GW strain for head-on white dwarf$\mathrm{BH}$ collisions with $M_{*}=M_{\odot}$ and various values of $M_{\mathrm{BH}}$, assumed to be observed with optimal orientation at a distance of $d=1 \mathrm{Mpc}$. For comparison, we also show the Advanced LIGO and KAGRA noise curve.

the $\mathrm{S} / \mathrm{N}$ by $\sim 70 \%$ (this doubles the overall amplitude, but shifts the signal to lower frequencies where the sensitivity is somewhat lower), while scaling to $M_{*}=1 M_{\odot}$ roughly halves the $\mathrm{S} / \mathrm{N}$.

The frequency of the GWs produced by the white dwarf- $\mathrm{BH}$ systems considered here would fall at higher frequencies, outside the range of a LISA-like instrument. The ringdown of $\mathrm{BHs}$ with masses $\lesssim 10^{3} M_{\odot}$ will occur in the Advanced LIGO sensitivity range; however, though those with higher masses will fall in the intermediate regime where neither instrument is sensitive. Gravitational wave detectors utilizing atom interferometers could potentially fill this frequency gap (Dimopoulos et al. 2008), as could other proposed space-based laser interferometers such as DECIGO (Kawamura et al. 2006) or BBO (Phinney et al. 2003). In Figure 13, we show the characteristic GW strain from the white-dwarf simulations, assuming $M_{*}=M_{\odot}$ and observation with optimal orientation at a distance of $d=1 \mathrm{Mpc}$. For comparison, we also show the proposed broadband Advanced LIGO noise curve (Shoemaker 2011) and the broadband KAGRA noise curve (LCGT Special Working Group 2009). We can see that for this particular choice of physical mass, the strongest signal occurs for $M_{\mathrm{BH}} / M_{*}=1$ to $2 \times 10^{3}(\mathrm{~S} / \mathrm{N} \approx 3$ for the KAGRA noise curve) since for higher $\mathrm{BH}$ mass the frequency of the signal is too low, and for lower masses, tidal effects strongly suppress the GW signal. The curves shown are all for head-on collisions, though, of course, generically the collision will occur with angular momentum. As mentioned in Section 5. the geodesic model for the $M_{\mathrm{BH}} / M_{*}=2 \times 10^{3}$ mass ratio suggests a collision with $\tilde{L} / M_{\mathrm{BH}} \sim 2$ should be similarly coherent as the head-on case, thus angular momentum could boost the detectability by a factor of a few. A collision with a spinning BH would also be somewhat easier to detect because of the extra power at high frequencies.

\section{CONCLUSION}


In this paper, we explored the GWs produced by collisions of strongly tidally affected stars with massive BHs. Taking into account the dynamics of the tidally perturbed star collision process and the distribution of the star's mass, we found that nonnegligible GWs are produced well into the regime where the star is colliding with the $\mathrm{BH}$ over a timescale comparable to the period of the GWs. We also found that collisions with angular momentum can substantially boost the GW strength, as can BH spin. The main features of our results were wellcaptured by a simple model based on geodesics.

For main-sequence stars and BHs with $M_{\mathrm{BH}} \geq 10^{6} M_{*}$, we found that for most of the range of merging values of angular momentum, the waveform is still similar to that of a point particle. Tidal disruption event rates per galaxy are estimated to be roughly in the range of $\sim 10^{-5}-10^{-3} \mathrm{yr}^{-1}$ (Wang \& Merritt 2004, Gezari et al. 2008, van Velzen et al. 2011; Kesden 2012) and to vary inversely with BH mass. The results found here suggest that collisions occurring in the Virgo cluster, which contains $\sim 10^{3}$ galaxies, could be visible by a $L I S A$-like $\mathrm{GW}$ detector for a range of parameters. While event rates involving intermediate-mass BHs are not well-known, our results also suggest that there is a small window of parameter space for a white dwarf collision where the $\mathrm{BH}$ is not so massive that the ringdown signal falls outside the frequency range of a ground-based detector, but still not small enough in mass that decoherence completely suppresses the gravitational radiation. While these sources will most likely not have very high rates for near-future GW detectors, these results do illustrate that such events can be direct probes of the BH's characteristics if they occur sufficiently close, or if they are targeted by a subsequent generation of detectors.

Though neutron stars have significantly higher compactions than the cases considered here, these results might also be used to inform an understanding of the GW signal produced when a neutron star merges with a stellar-mass $\mathrm{BH}$ and how tidal effects may suppress the merger-ringdown signal. For future study, it would also be interesting to explore high-angular-momentum collisions with spinning BHs since the point-particle prediction suggests that spin could enhance the strength of the GW signal significantly Kojima \& Nakamura 1983a). In upcoming work, we will use these same numerical methods to explore noncollisional tidal disruption events (W. E. East \& F. Pretorius 2014, in preparation).

I thank Frans Pretorius and Nick Stone for valuable comments on this work. Simulations were run on the Bullet cluster at SLAC and the Orbital cluster at Princeton University.

\section{REFERENCES}

Abramovici, A., Althouse, W. E., Drever, R. W., Gursel, Y. Kawamura, S., et al. 1992, Science, 256, 325

Alcubierre, M., Brandt, S. R., Brügmann, B., Holz, D. E., Seidel, E., Takahashi, R., \& Thornburg, J. 2001, Int. J. Mod. Phys. D, 10, 273, arXiv:gr-qc/9908012

Amaro-Seoane, P. et al. 2013, GW Notes, Vol. 6, p. 4-110, 6, 4, 1201.3621

Amaro-Seoane, P., Gair, J. R., Freitag, M., Miller, M. C., Mandel, I., Cutler, C. J., \& Babak, S. 2007, Classical and Quantum Gravity, 24, 113, astro-ph/0703495

Bade, N., Komossa, S., \& Dahlem, M. 1996, A\&A, 309, L35

Berti, E., Cardoso, V., \& Will, C. M. 2006, Phys. Rev. D, 73, 064030, arXiv:gr-qc/0512160

Bloom, J. S. et al. 2011, Science, 333, 203, 1104.3257

Cappelluti, N. et al. 2009, A\&A, 495, L9, 0901.3357

Cenko, S. B. et al. 2012a, MNRAS, 420, 2684, 1103.0779

. 2012b, ApJ, 753, 77, 1107.5307

Cheng, R. M., \& Evans, C. R. 2013, Phys. Rev. D, 87, 104010 , 1303.4129

Cook, G. B., \& Scheel, M. A. 1997, Phys. Rev. D, 56, 4775

Cook, G. B., Shapiro, S. L., \& Teukolsky, S. A. 1994, ApJ, 422, 227

Davis, M., Ruffini, R., Press, W. H., \& Price, R. H. 1971, Phys. Rev. Lett., 27, 1466

Dimopoulos, S., Graham, P. W., Hogan, J. M., Kasevich, M. A. \& Rajendran, S. 2008, Phys. Rev. D, 78, 122002, 0806.2125

East, W. E., \& Pretorius, F. 2013, Phys.Rev., D87, 101502, 1303.1540

East, W. E., Pretorius, F., \& Stephens, B. C. 2012a, Phys.Rev., D85, 124010, 1112.3094

East, W. E., Ramazanoglu, F. M., \& Pretorius, F. 2012b, Phys. Rev., D86, 104053, 1208.3473

Frank, J., \& Rees, M. J. 1976, MNRAS, 176, 633

Gezari, S. et al. 2008, ApJ, 676, 944, 0712.4149

- 2012, Nature, 485, 217, 1205.0252

Gezari, S., Halpern, J. P., Komossa, S., Grupe, D., \& Leighly, K. M. 2003, ApJ, 592, 42

Gezari, S. et al. 2006, ApJ, 653, L25, arXiv:astro-ph/0612069

Guillochon, J., Ramirez-Ruiz, E., Rosswog, S., \& Kasen, D. 2009 ApJ, 705, 844, 0811.1370

Haas, R., Shcherbakov, R. V., Bode, T., \& Laguna, P. 2012, ApJ, $749,117,1201.4389$
Haugan, M. P., Shapiro, S. L., \& Wasserman, I. 1982, ApJ, 257, 283

Irwin, J. A., Brink, T. G., Bregman, J. N., \& Roberts, T. P. 2010, ApJ, 712, L1, 0908.1115

Kawamura, S. et al. 2006, Classical and Quantum Gravity, 23, 125

Kesden, M. 2012, Phys. Rev. D, 85, 024037, 1109.6329

Kobayashi, S., Laguna, P., Phinney, E. S., \& Mészáros, P. 2004, ApJ, 615, 855, astro-ph/0404173

Kojima, Y., \& Nakamura, T. 1983a, Physics Letters A, 99, 37

1983b, Physics Letters A, 96, 335

Komossa, S., \& Greiner, J. 1999, A\&A, 349, L45, arXiv:astro-ph/9908216

Krolik, J. H., \& Piran, T. 2011, ApJ, 743, 134, 1106.0923

Larson, S. L. accessed June 2014, Online Sensitivity Curve Generator, http://www.srl.caltech.edu/\%7Eshane/sensitivity/

LCGT Special Working Group. 2009, KAGRA official sensitivity limit,

http://gwcenter.icrr.u-tokyo.ac.jp/en/researcher/parameter

Levan, A. J. et al. 2011, Science, 333, 199, 1104.3356

MacLeod, M., Goldstein, J., Ramirez-Ruiz, E., Guillochon, J., \& Samsing, J. 2014, ApJ, 794, 9, 1405.1426

Nakamura, T., \& Sasaki, M. 1982, Physics Letters A, 89, 185

Phinney, E. S., et al. 2003, The Big Bang Observer: direct detection of gravitational waves from the birth of the Universe to the present, Tech. rep., Washington, DC

Pretorius, F. 2005, Class. Quantum Grav., 22, 425, arXiv:gr-qc/0407110

Shcherbakov, R. V., Pe'er, A., Reynolds, C. S., Haas, R., Bode, T., \& Laguna, P. 2013, ApJ, 769, 85, 1212.4837

Shoemaker, D. 2011, Advanced LIGO Anticipated Sensitivity Curves, https://dcc.ligo.org/cgi-

bin/DocDB/ShowDocument?docid $=2974$

Somiya, K. 2012, Classical and Quantum Gravity, 29, 124007 , 1111.7185

Stone, N., Sari, R., \& Loeb, A. 2013, MNRAS, 435, 1809, 1210.3374

van Velzen, S. et al. 2011, ApJ, 741, 73, 1009.1627

Wang, J., \& Merritt, D. 2004, ApJ, 600, 149, astro-ph/0305493

Zalamea, I., Menou, K., \& Beloborodov, A. M. 2010, MNRAS, 409, L25, 1005.3987

Zauderer, B. A. et al. 2011, Nature, 476, 425, 1106.3568 